\title{
Computable Functions, Quantum Measurements, and Quantum Dynamics
}

\author{
M. A. Nielsen* \\ Center for Advanced Studies, Department of Physics and Astronomy, University of New Mexico, \\ Albuquerque, New Mexico 87131-1156 \\ and Norman Bridge Laboratory of Physics 12-33, California Institute of Technology, Pasadena, California 91125
}

(Received 4 June 1997)

\begin{abstract}
We construct quantum mechanical observables and unitary operators which, if implemented in physical systems as measurements and dynamical evolutions, would contradict the Church-Turing thesis which lies at the foundation of computer science. We conclude that either the Church-Turing thesis needs revision, or that only restricted classes of observables may be realized, in principle, as measurements, and that only restricted classes of unitary operators may be realized, in principle, as dynamics. [S0031-9007(97)04263-4]

PACS numbers: $89.70 .+\mathrm{c}, 03.65 . \mathrm{Bz}$
\end{abstract}

Quantum mechanical measurements on a physical system are represented by observables - Hermitian operators on the state space of the observed system. It is an important question whether all observables may be realized, in principle, as measurements on a physical system. Dirac's influential text [1] makes the following assertion on the question:

The question now presents itself - can every observable be measured? The answer theoretically is yes. In practice it may be very awkward, or perhaps even beyond the ingenuity of the experimenter, to devise an apparatus which could measure some particular observable, but the theory always allows one to imagine that the measurement can be made.

This Letter reexamines the question of whether it is possible, even in principle, to measure every quantum mechanical observable. Unexpectedly, ideas from computer science are crucial to the analysis. We also investigate a related question, namely, whether it is possible to realize, in principle, the dynamics corresponding to an arbitrary unitary operator on the state space of a quantum system. Of course, for specific systems particular measurements and unitary dynamics may be forbidden by system specific features, such as superselection rules. We will not be concerned with such specific features, but rather with general considerations.

In the remarkable paper which founded modern computer science, Turing [2] defined a class of functions which are now known as the recursive or computable functions. The Church-Turing thesis [2,3] of computer science states that this class of functions corresponds precisely to the class of functions which may be computed via what humans intuitively call an algorithm or procedure. More formally, every function which can be computed by what we would naturally regard as an algorithm is a computable function, and vice versa.

The Church-Turing thesis is fundamental to theoretical computer science, since it asserts that the mathematical class of functions studied by computer scientists, the com- putable functions, is the most general class of functions which may be calculated using a computer. It is an empirical statement, not a theorem of mathematics, and has been verified through over 60 years of testing $[4,5]$. For a review of different formulations of the Church-Turing thesis, see [4].

For convenience, we refer to computable partial functions of a single non-negative integer as programs. That programs are only partial functions means that there may be some values of the input for which no output is defined. An example of a program is the function $f(x)=x^{2}$, which may be computed using a suitable Turing machine. Using Turing's results, it is possible to show [6] that the set of programs may be numbered $0,1,2,3, \ldots$ Not all programs need to terminate or halt for all possible inputs. A simple example is the program $f$ which on input $x$ loops forever if $x$ is not a perfect square, or prints $\sqrt{x}$ if $x$ is a perfect square. This is an example of a partial function.

The question of whether or not a given program, numbered $x$, halts on the input of $y$ is obviously a question of considerable practical importance: we would like to know whether a given algorithm will terminate or not. To understand this question better, Turing defined a function, the halting function $h$, by

$$
h(x) \equiv \begin{cases}1 & \text { if program } x \text { halts on input } x \\ 0 & \text { if program } x \text { does not halt on input } x\end{cases}
$$

Turing [2] demonstrated that the halting function is not a computable function. That is, there exists no algorithmic means for computing the value of the halting function for all values $x=0,1, \ldots$. Thus there is no algorithm for determining whether a given program terminates or not.

Many noncomputable functions other than the halting function are now known, and the reasoning which follows applies to any such function $h$. For concreteness we continue to imagine that $h$ is the halting function. 
We define the halting observable, $\hat{h}$, by

$$
\hat{h} \equiv \sum_{x=0}^{\infty} h(x)|x\rangle\langle x|,
$$

where $|x\rangle$ is an orthonormal basis for the state space of some physical system with a countably infinite dimensional state space. We will suppose that the system is one such that all the states $|x\rangle$ may be prepared, in principle. For example, the states $|x\rangle$ might be number states of a single mode of the electromagnetic field. The halting observable is clearly an observable in the usual quantum mechanical sense: it is a Hermitian operator on the state space of the system of interest.

Logically, one of two possibilities must hold:

(1) It is possible, in principle, to construct a measuring device capable of performing a measurement of the observable $\hat{h}$.

(2) It is not possible, in principle, to construct a measuring device capable of performing a measurement of the observable $\hat{h}$.

Suppose the first possibility is true. Then in order to compute the value of $h(x)$ one performs the following procedure: Construct the measuring apparatus to measure $\hat{h}$, and prepare the system to be measured in the state $|x\rangle$. Now perform the measurement. With probability one the result of the measurement will be $h(x)$. This gives a procedure for computing the halting function. If one accepts the Church-Turing thesis this is not an acceptable conclusion, since the halting function is not computable.

Acceptance of the Church-Turing thesis therefore forces us to conclude that the second option is true, namely, that it is not possible, in principle, to construct a measuring device capable of performing a measurement of the observable $\hat{h}$. That is, only a limited class of observables correspond to measurements which may be performed, in principle, on quantum mechanical systems. An important question arises: to determine the precise class of observables which may be realized as measurements.

Might it be possible to perform an approximate measurement of $\hat{h}$ ? Suppose it is possible to measure an observable $\hat{h}^{\prime}$ which is close to $\hat{h}$. Preparing the system in the state $|x\rangle$ and measuring $\hat{h}^{\prime}$, a result in the range $h(x) \pm \delta$ is obtained with probability at least $1-\epsilon$, for some small $\epsilon$ and $\delta$. Clearly, by performing repeated measurements of this type it is possible to determine $h(x)$ with arbitrarily high confidence. Thus, approximate measurements of $\hat{h}$ give an algorithmic means for computing $h(x)$. Once again, if we accept the Church-Turing thesis, then we are forced to conclude that it is not possible to perform such an approximate measurement. Note, however, that we are implicitly using a stronger version of the Church-Turing thesis than hitherto, since now we are regarding as an algorithm a procedure which outputs $h(x)$ with arbitrarily high confidence, rather than a purely deterministic procedure.
This second conclusion should be compared to work by Wigner [7], and Araki and Yanase [8] on the WignerAraki-Yanase (WAY) theorem [9]. The WAY theorem shows that if we require certain conservation laws to be respected during the measurement process, then there are restrictions on the class of observables which may be realized using measuring devices. This is fundamentally different to the conclusion we have obtained, which does not depend on the imposition of externally imposed conservation requirements. Another difference is that it was shown in [8] and [10] that it is possible to perform approximate measurements of the observables forbidden realization as measurements by the WAY theorem. As we have already seen, approximate measurements of $\hat{h}$ are not allowed by the Church-Turing thesis.

Up to this point, we have considered the physical realization of measurements corresponding to quantum mechanical observables. Similar arguments apply also to the physical realization of unitary operators as dynamical evolutions. Define a function $g$ as follows:

$$
g(x) \equiv \begin{cases}2 m-2 & \text { if } x \text { is the } m \text { th smallest non-negative } \\ & \text { integer such that } h(x)=0, \\ 2 m-1 & \text { if } x \text { is the } m \text { th smallest non-negative } \\ & \text { integer such that } h(x)=1 .\end{cases}
$$

It is easy to verify that the operator

$$
U \equiv \sum_{x=0}^{\infty}|g(x)\rangle\langle x|
$$

is unitary. Suppose we prepare the system in the state $|x\rangle$, perform the unitary dynamics $U$, and then do a measurement in the $|x\rangle$ basis with outcome $x^{\prime}$ (note that there are systems where such a measurement can certainly be done, in principle, such as a single mode of the electromagnetic field). Note that $x^{\prime}$ is even if and only if $h(x)=0$ and $x^{\prime}$ is odd if and only if $h(x)=1$, so this gives a procedure for computing the halting function. Once again, logically, one of two possibilities must hold:

(1) It is possible, in principle, to construct a system whose dynamics is described by the unitary operator $U$.

(2) It is not possible, in principle, to construct a system whose dynamics is described by the unitary operator $U$. Once again, if we accept the Church-Turing thesis, then we are forced to the second conclusion: there are unitary operators which do not describe the dynamics of any system which can, even in principle, be constructed. By arguments similar to those used for observables, it is easy to see that an approximate dynamical realization of $U$ can also be used as part of a procedure for evaluating the halting function, so acceptance of the Church-Turing thesis implies that approximate realizations of $U$ are not possible, either.

The examples we have discussed take place in infinite dimensional state spaces. A similar construction for a spin $\frac{1}{2}$ system starts by defining (see chapter seven of 
[11] for a review of definitions along these lines, and references)

$$
\Omega \equiv \sum_{x: h(x)=1} \frac{1}{2^{x}} .
$$

Note that $0<\Omega<1$, and that the $x$ th bit in the binary expansion of $\Omega$ is one if and only if $h(x)=1$, so knowing the binary expansion of $\Omega$ is equivalent to knowing $h(x)$ for all $x$. Define $U \equiv \exp \left(-i \Omega \sigma_{y}\right)$. Starting the system in the $\left|\frac{1}{2}, \frac{1}{2}\right\rangle$ state (spin up in the $z$ direction) and applying the dynamics $U$ we see that the state after the dynamics is

$$
\cos (\Omega)\left|\frac{1}{2}, \frac{1}{2}\right\rangle+\sin (\Omega)\left|\frac{1}{2},-\frac{1}{2}\right\rangle \text {. }
$$

By repeatedly performing this procedure and making measurements of $\sigma_{z}$ we may determine $\cos (\Omega)$ and thus $\Omega$ to any desired accuracy, with arbitrarily high confidence. It follows that we can determine the value of $h(x)$ for all $x$. Once again, if we accept the Church-Turing thesis, then we are forced to conclude that $U$ cannot be realized. Notice that, unlike the earlier examples, this procedure is not stable under perturbations of $U$. A slight change in $U$ can result in an incorrect evaluation of $h(x)$. Physically, uncontrolled interactions with the environment will necessarily mean that $U$ is not implemented exactly, and thus it is not possible to evaluate the halting function using a dynamical realization of $U$. Based on similar arguments it seems likely, though I know of no rigorous general proof, that any finite dimensional construction which allows evaluation of a noncomputable function is unstable against perturbations, and therefore is not physically interesting.

Returning to the two physically interesting infinite dimensional examples, what conclusions can be drawn? There are two programs one might pursue.

The first program is to modify the Church-Turing thesis. Perhaps there exist in nature quantum processes which can be used to compute functions which are classically noncomputable. It is far-fetched, but not logically inconsistent, to imagine some type of experimentperhaps a scattering experiment-which can be used to evaluate the halting function.

Recognizing such a process poses some problems. How could we verify that a process computes the halting function (or any other noncomputable function)? Because of the unsolvability of the halting problem, it is not possible to verify directly that the candidate "halting process" is, in fact, computing the halting function. Nevertheless, one can imagine inductively verifying that the process computes the halting function. One would do this by running a large number of programs on a computer for a long time, and checking that all the programs which halt are predicted to halt by the candidate halting process, and that programs predicted not to halt by the candidate halting process have not halted. Given sufficient empirical evidence of this sort, one could then postulate as a new physical law that the process computes the halting function.

What types of modification of the Church-Turing thesis might be considered in this program? One approach is to exclude quantum phenomena by fiat from the area of application of the thesis. Approaches of this type have numerous problems. First, the boundary between quantum and classical phenomena is rather fuzzy; where precisely does one draw the line? Second, the approach is ad hoc; what motivates the rejection of quantum phenomena from the area of application of the ChurchTuring thesis? Many other modifications of the ChurchTuring might be attempted, however, we will not discuss them here, as no fully satisfactory modification has been found by the author.

It is the author's conjecture that the Church-Turing thesis is essentially correct, and that a more satisfactory program is to address the problem of achieving a sharp characterization of the class of observables and unitary dynamics which may be realized in physical systems. At least two properties must be satisfied by such a characterization:

(1) It should be consistent with a (possibly sharpened) form of the Church-Turing thesis.

(2) It should be clear that the measurements and dynamics contained in that class are, in principle, realizable, and that all other measurements and dynamics could never be realized, even in principle, in the laboratory.

How might one achieve such a characterization? Deutsch [12] has proposed what he calls the ChurchTuring principle, to distinguish it from the less well formulated Church-Turing thesis. The statement of this principle reads:

Every finitely realizable physical system can be perfectly simulated by a universal model computing machine operating by finite means.

Note that finite means here has the meaning that on any given computation finite computational resources are used. As for classical computers, unbounded resources are in principle available, provided only finite resources are used on any given computation. Finitely realizable is being used in the same sense as we have used realizable: in principle, the system can be constructed in a laboratory.

Given such a universal machine, the problem of studying what classes of measurements and dynamics are realizable is reduced to the study of properties of a single physical system, the universal computing machine, since that system can be used to simulate all other finitely realizable physical systems. This reduction to studying the properties of a single system may make it considerably easier to study the classes of measurements and dynamics which are realizable.

For example, Deutsch showed that his proposed universal computing machine cannot compute any function which is not computable on a classical computer. Thus, his machine cannot compute the halting function. But, 
if we assume that the halting observable can be measured using some physical system, then by the ChurchTuring principle the measurement could be simulated on a universal computing machine, and the result of the measurement determined. This contradicts Deutsch's assertion that such a machine cannot compute any function which is not computable classically, and we conclude that measurements of the halting observable are not possible, in principle. Thus, if we accept that Deutsch's proposed machine satisfies the Church-Turing principle, then it follows that the halting observable cannot be measured.

What is gained by using arguments based on the Church-Turing principle instead of arguments based on the Church-Turing thesis, is that it may be possible to prove the Church-Turing principle within known physical theory, for a suitable universal model computing machine. Unfortunately, it is not clear to this author that the theory of quantum computation (see [13-15] for a review), which has developed from Deutsch's original proposal, provides a candidate universal model computing machine. In particular, it is not clear that the finite dimensional state spaces accessed by quantum computers are sufficient to simulate, with arbitrary accuracy, all the processes one finds in nature. Natural processes may take place in infinite dimensional state spaces, and it is has not been demonstrated that all such processes can be well simulated using a system with only a finite number of state space dimensions. Regardless of whether quantum computers satisfy the Church-Turing principle, it is certainly the case that the specification of a universal model computing machine satisfying the Church-Turing principle, besides being important in its own right, would also greatly simplify the question of characterizing the classes of measurements and dynamics which are realizable in physical systems.

This Letter has discussed two questions: what class of observables may be realized as quantum measurements; and what unitary operators may be realized as quantum dynamics. Using concepts from computer science we have constructed observables and unitary operators whose physical implementation would contradict the fundamental Church-Turing thesis of computer science. We con- clude that the introduction of new concepts into computer science, physics, or both, is necessary to resolve this contradiction.

I thank Tony Bracken, Ike Chuang, Phil Diamond, HoiKwong Lo, Gerard Milburn, John Preskill, and Howard Wiseman for discussions about this paper. This work began with the support of a 1993 summer vacation scholarship in the University of Queensland Mathematics Department, and was continued with the support of the Office of Naval Research (Grant No. N00014-93-1-0116), the support of DARPA through the Quantum Information and Computing (QUIC) institute administered by the Army Research Office, and the Australian-American Educational Foundation (Fulbright Commission).

*Electronic address: mnielsen@tangelo.phys.unm.edu

[1] P.A.M. Dirac, The Principles of Quantum Mechanics (Oxford University Press, Oxford, 1958), 4th ed., p. 37.

[2] A. M. Turing, Proc. Lond. Math. Soc. 2 42, 230 (1936) (reprinted in [16]).

[3] A. Church, Am. J. Math. 58, 345 (1936) (reprinted in [16]).

[4] D. R. Hofstadter, Gödel, Escher, Bach: An Eternal Golden Braid (Basic Books, New York, 1979).

[5] R. Penrose, The Emperor's New Mind (Oxford University Press, Oxford, 1989).

[6] M. D. Davis and E. J. Weyuker, Computability, Complexity, and Languages (Academic Press, New York, 1983).

[7] E. P. Wigner, Z. Phys. 133, 101 (1952).

[8] H. Araki and M. M. Yanase, Phys. Rev. 120, 622 (1960).

[9] A. Peres, Quantum Theory: Concepts and Methods (Kluwer Academic, Dordrecht, 1993).

[10] G. C. Ghirardi, F. Miglietta, A. Rimini, and T. Weber, Phys. Rev. D 24, 347 (1981).

[11] T. M. Cover and J. A. Thomas, Elements of Information Theory (John Wiley and Sons, New York, 1991).

[12] D. Deutsch, Proc. R. Soc. Lond. A 400, 97 (1985).

[13] A. Ekert and R. Jozsa, Rev. Mod. Phys. 68, 1 (1996).

[14] D. P. DiVincenzo, Science 270, 255 (1995).

[15] C. H. Bennett, Phys. Today 48, No. 10, 255 (1995).

[16] M.D. Davis, The Undecidable (Raven Press, Hewlett, New York, 1965). 